\title{
Giant optical birefringence in ensembles of semiconductor nanowires
}

\author{
O. L. Muskens \\ FOM Institute for Atomic and Molecular Physics AMOLF, c/o Philips Research Laboratories, \\ High Tech Campus 4, 5656 AE, Eindhoven, The Netherlands \\ M. T. Borgström and E. P. A. M. Bakkers \\ Philips Research Laboratories, High Tech Campus 4, 5656 AE Eindhoven, The Netherlands \\ J. Gómez Rivas ${ }^{\text {a) }}$ \\ FOM Institute for Atomic and Molecular Physics AMOLF, c/o Philips Research Laboratories, \\ High Tech Campus 4, 5656 AE Eindhoven, The Netherlands
}

(Received 8 August 2006; accepted 27 October 2006; published online 7 December 2006; corrected 15 December 2006)

\begin{abstract}
Giant birefringence is demonstrated in ensembles of randomly grown, vertically aligned semiconductor nanowires. Bottom-up fabrication of epitaxial semiconductor nanowires grown using the vapor-liquid-solid mechanism yields a highly anisotropic photonic metamaterial. The birefringence in the material is shown to depend on the nanowire length. Short nanowires with a volume filling fraction around $50 \%$ exhibit the strongest birefringence, in agreement with effective medium theories. A large difference is found between the in-plane and out-of-plane refractive indices of $\Delta n=0.8$, exceeding by a factor of 75 the natural birefringence of quartz and by more than a factor of 2 that of inverted artificial materials. (C) 2006 American Institute of Physics.
\end{abstract}

[DOI: 10.1063/1.2402906]

Semiconductor nanowires are nanostructures with a diameter much smaller and a length much larger than the wavelength of visible light. This large anisotropy, combined with the high refractive index of some semiconductors, leads to fascinating optical phenomena. Giant polarization anisotropy for the absorption and emission of light was first reported by Wang et al. ${ }^{1}$ by measuring the photoluminescence of individual InP nanowires. It was demonstrated that semiconductor nanowires can function as polarization sensitive photodectectors. ${ }^{1,2}$ Furthermore, the polarization anisotropy of the optical properties of nanowires has been used to selectively photopassivate their surface. ${ }^{3}$ Very recently, we demonstrated that nanowires exhibit form birefringence, with an optical axis that can be controlled by the direction of the epitaxial growth. ${ }^{4}$

Optical birefringence describes the difference in refractive index of light with different polarizations traveling inside an anisotropic material. Next to the natural birefringence of crystals, artificial birefringence occurs in materials containing aligned macroscopic scatterers. The phenomenon has been observed in various systems such as aligned carbon nanotube films, ${ }^{5}$ porous silicon, ${ }^{6,7}$ and liquid-crystal templated porous $\mathrm{CdTe}^{8}$ In this letter we show that giant birefringence can be achieved in dense collections of randomly positioned vertical nanowires by controlling their length, alignment and diameter, and thereby the semiconductor filling fraction. The largest birefringent ensemble that we have measured has an extraordinary large birefringence of 0.8 , exceeding the natural birefringence of quartz by a factor of 75 and that of inverted or porous materials by more than a factor of 2. ${ }^{6,7}$ This is to our knowledge the strongest birefringent material to date based on randomly positioned scatterers in the long-wave limit.

\footnotetext{
${ }^{a}$ Electronic mail: rivas@amolf.nl; URL: http://www.nanowirephotonics. com
}

The semiconductor nanowire materials of this study are extremely suitable for several important technological applications involving porous media, such as optical biosensing. ${ }^{7,9,10}$ The extremely large available surface area of the wires can be coated with additional layers of silicon or noble metals for compatibility with biological functionalization groups, ${ }^{9,11}$ while local bottom-up growth of nanowire layers on optical waveguides will yield integrated optical sensors with improved sensitivity. ${ }^{10}$ Other applications of giant-birefringence nanowire materials are anticipated in quantum optics as a source of entangled photons, ${ }^{12}$ for polarization control in microphotonics, ${ }^{13}$ and as propagation medium for surface waves on birefringent materials. ${ }^{14}$

We have investigated layers of different thicknesses of vertically oriented $\mathrm{GaP}$ nanowires grown in a high volume density of several percent. GaP has a large refractive index $n_{\mathrm{GaP}}$ of 3.3 in the red part of the visible spectrum and an energy band gap of $2.26 \mathrm{eV}{ }^{15}$ The nanowires were grown with the vapor-liquid-solid ${ }^{16}$ method by the use of metalorganic vapor phase epitaxy at $420{ }^{\circ} \mathrm{C}$ on a (111)B GaP substrate. A thin $(0.2 \mathrm{~nm})$ gold film was deposited on the substrate, resulting at the deposition temperature in a high density of individual catalyst droplets from which the nanowires are grown. The thickness of the nanowire layer was controlled by the growth time.

Figures 1(a)-1(c) show cross-sectional scanning electron microscopy (SEM) images of three nanowire layers of different lengths, epitaxially grown on a GaP substrate. The semiconductor filling fraction in the three samples is approximately $4 \%$. The scale bars in the three images correspond to $1 \mu \mathrm{m}$. Figure 1(a) represents a layer of nanowires with a length of $14.5 \mu \mathrm{m}$, while the nanowires of Figs. 1(b) and 1(c) have lengths of about 4.7 and $1.5 \mu \mathrm{m}$. For all samples the nanowires were epitaxially grown in the vertical (111) $B$ direction; however, with increasing length, the wires seem to bend due to their increasing flexibility. This phenom- 

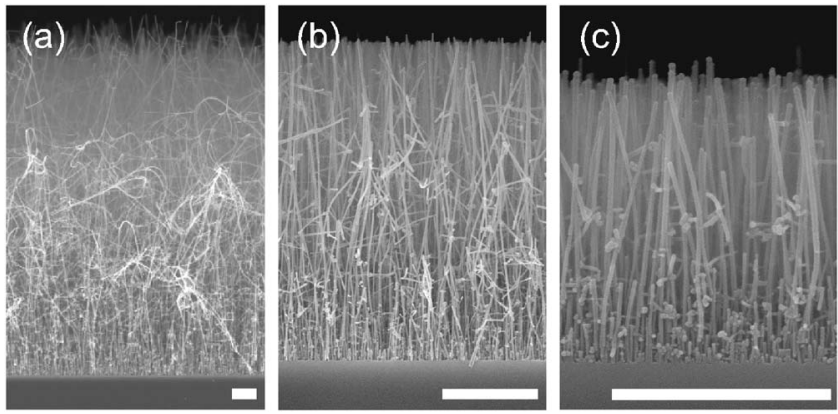

FIG. 1. Cross-sectional SEM images of GaP nanowires epitaxially grown on a GaP substrate. (a), (b), and (c) correspond to layers of nanowires with lengths of $14.5,4.7$, and $1.5 \mu \mathrm{m}$, respectively. The scale bar represents $1 \mu \mathrm{m}$ in all the figures.

enon, as we will see, is responsible for a reduction of the birefringence.

For wires oriented perpendicular to the surface, the optical birefringence is expected to vary strongly between normal and in-plane incidence of light. The nanowire material may be considered as a positively uniaxial medium with corresponding values for the refractive indices $n_{o}$ and $n_{e}$, respectively, for polarizations perpendicular and parallel to the nanowire axes. The values for $n_{o}$ and $n_{e}$ can be calculated from a microscopic effective medium model reflecting the specific geometrical distribution of the mixture. For a material consisting of perfectly aligned cylindrical pillars, it has been shown theoretically that in the long-wavelength limit the two indices are described by the Maxwell-Garnett effective medium model for the ordinary index $n_{o}$ and the geometrical average for extraordinary refractive index $n_{e} \cdot{ }^{17-19} \mathrm{In}$ the case of GaP pillars of refractive index $n_{\mathrm{GaP}}$ occupying a volume fraction $f$ in air, we have

$$
\begin{aligned}
& n_{o}^{2}=\left(1+\frac{2 f \alpha}{1+f \alpha}\right), \\
& n_{e}^{2}=f n_{\mathrm{GaP}}^{2}+(1-f),
\end{aligned}
$$

where $\alpha=\left(n_{\mathrm{GaP}}^{2}-1\right) /\left(n_{\mathrm{GaP}}^{2}+1\right)$ denotes the polarizability of the cylinder for an electric field perpendicular to the wire axis. This model describes the dielectric constants for a medium of cylinders and should hold as long as the wires are not overlapping.

To determine the material refractive indices, we use the method of angular-dependent polarization interferometry at an optical wavelength of $632.8 \mathrm{~nm}$ (He:Ne laser). The polarization of the input light beam is set to $45^{\circ}$ with respect to the plane of incidence. The rotation of the polarization vector after propagation through the nanowire layer is measured via the parallel $\left(I_{\|}\right)$and cross-polarized $\left(I_{\perp}\right)$ intensities (relative to the input polarization) using an analyzing polarization filter. The ratio of the intensities $I_{\perp} / I_{\|}$is a measure of the polarization extinction and represents the quality of the birefringent material. ${ }^{7}$ This ratio is shown in Fig. 2 as a function of incident angle $\theta_{\text {in }}$ for light transmitted through the three samples with increasing nanowire length of Fig. 1. For the sample with the longest nanowires a maximum at $57^{\circ}$ and a minimum at $78^{\circ}$ are observed in the polarization extinction (blue line in Fig. 2), corresponding to an optical retardation over multiple orders of $\pi$. The sample with $4.7 \mu \mathrm{m}$ long nanowires presents only a maximum at $72^{\circ}$ in the polarizaDownloaded 12 Jan 2007 to 192.87.154.163. Redistribution subject

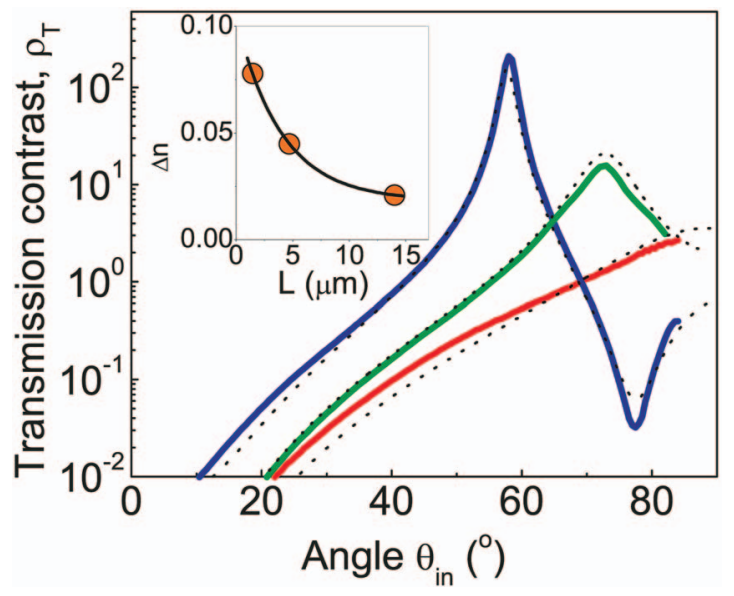

FIG. 2. (Color) Transmission contrast as a function of the angle of incidence $\theta_{\text {in }}$ for three layers of $\mathrm{GaP}$ nanowires with different thicknesses. The red line corresponds to a birefringent layer with a thickness of $1.5 \mu \mathrm{m}$, while the green and blue lines are samples with thicknesses of 4.7 and $14.5 \mu \mathrm{m}$, respectively. The dashed lines are fits to the measurements using the transfer matrix method. The inset represents the birefringence parameter of the samples obtained from the fits as a function of their thickness. The solid line is a guide for the eyes.

tion extinction ratio (green line in Fig. 2), while the sample with short nanowires (red line) does not have a maximum in the polarization extinction ratio due to the shorter optical path length in the birefringent layer.

Accurate estimates of $n_{e}$ and $n_{o}$ were obtained by fitting the polarization-dependent transmission using a transfer matrix model accounting for the various interfaces and for the birefringence of the nanowire layer. ${ }^{20}$ The results of these fits are represented by the dotted lines in Fig. 2 and show good quantitative agreement with the experimental results. The resulting birefringence coefficients $\Delta n=n_{e}-n_{o}$ for the three nanowire layers are plotted versus the layer thickness in the inset of Fig. 2. The solid line in this inset is a guide to the eyes. The birefringence coefficient increases strongly as the thickness of the layer is reduced. We attribute this to a better alignment of the short nanowires that do not show the pronounced bending experienced by the longer nanowires.

According to Eqs. (1) and (2) the birefringence coefficient can be increased by augmenting the semiconductor filling fraction $f$, reaching a maximum when $f$ is roughly $50 \%$. Therefore we have made samples of short nanowires with increasing semiconductor filling fractions by growing an additional $\mathrm{GaP}$ shell around the wires. This is done by elevating the growth temperature to $630{ }^{\circ} \mathrm{C}$ for a limited time after growing the nanowires. ${ }^{4}$ Figure 3 shows cross-sectional SEM images of three samples with increasing shell growth
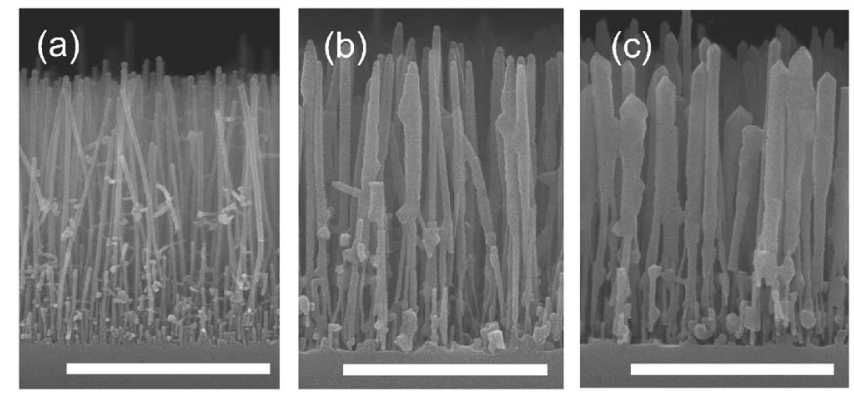

FIG. 3. Cross-sectional SEM images of GaP nanowire layers with radial

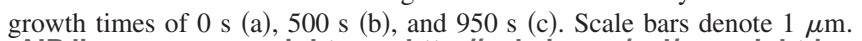
to AIP license or copyright, see http://apl.aip.org/apl/copyright.jsp 


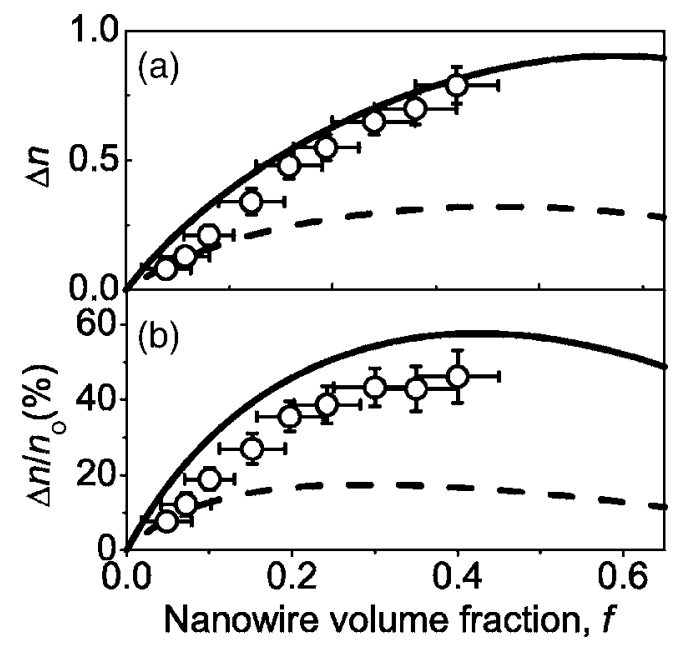

FIG. 4. (a) Birefringence $\Delta n$ against nanowire volume fraction $f$ for samples with an average nanowire length of $1.5 \mu \mathrm{m}$ and different radial growth times. The solid and dashed lines are the Maxwell-Garnett calculations [Eqs. (1) and (2)] for the direct (GaP pillars in air) and inverse medium (pores in GaP). (b) Relative birefringence $\Delta n / n_{o}$ vs the nanowire volume fraction, with (lines) Maxwell-Garnett calculations.

times of (a) $0 \mathrm{~s}$, (b) $500 \mathrm{~s}$, and (c) $950 \mathrm{~s}$. The radial growth of $\mathrm{GaP}$ on the sidewalls of the nanowires results in an increase of the average nanowire diameter from (a) $21.5 \mathrm{~nm}$, (b) $45 \mathrm{~nm}$, up to (c) $90 \mathrm{~nm}$. The average volume density of nanowires, denoted as $f$, was determined from gravimetrical measurements and top-view SEM images to $f<0.04$ for the material without shell up to $f=0.40 \pm 0.05$ for the sample with the longest radial growth time.

The resulting birefringence coefficients $\Delta n$ for the samples with increasing radial growth times are plotted versus the semiconductor volume fraction $f$ in Fig. 4(a) (open circles) ${ }^{20}$ The birefringence increases strongly with the volume fraction. We obtain values as high as $0.80 \pm 0.07$ for a filling fraction of $40 \%$. The solid line in Fig. 4(a) represents the theoretical value for the birefringence coefficient using Eqs. (1) and (2). There is a remarkable good agreement between the theoretical and experimental values. The small discrepancy could be caused by the aforementioned bending of the nanowires, which yields a lower $\Delta n$ than that predicted by the theoretical model of perfectly aligned nanowires. ${ }^{18}$ For comparison we have also plotted in Fig. 4(a) the birefringence coefficient for an inverted structure consisting of air pores in a matrix of high dielectric material (dashed line). These types of structures are usually obtained by top-down fabrication methods. High density ensembles of nanowires present a much higher birefringence than inverted materials. The value of $\Delta n=0.80$ measured in $\mathrm{GaP}$ nanowires is more than a factor 2 higher than previously reported values in highly birefringent porous $\mathrm{Si}^{6,7}$

A parameter of considerable interest is the birefringence normalized to the in-plane refractive index $n_{o}$, describing the relative difference of the phase velocity of the light between the two polarizations. We have represented this relative birefringence in Fig. 4(b) as a function of the semiconductor filling fraction $f$. The relative birefringence reaches up to a value of $43 \%$. The large relative birefringence again is specific for the nanowire material, due to the very strong suppression of the nanowire polarizability perpendicular to its axis, ${ }^{1}$ and very different from porous network materials [dashed line in Fig. 4(b)].

In conclusion, we have fabricated a novel metamaterial based on high densities of semiconductor nanowires. The material shows large optical birefringence depending on the length of the nanowires. Short nanowires $(1.5 \mu \mathrm{m}$ long $)$ with a filling fraction of $40 \%$ yield a birefringence of $\Delta n=0.8$, two times larger than previously reported values of birefringence in inverted material such as porous films. The good quantitative agreement of these measurements with the theoretically calculated maximum birefringence demonstrates the high quality achieved in the fabrication of the nanowire materials in this work. The unique properties of the giantbirefringence nanowire materials combined with their local bottom-up fabrication open up important technological applications in optical sensing and nanophotonics.

The authors thank George Immink, Eddy Evens, Frans Holthuysen, and Peter Breijmer for technical assistance and Gert 't Hooft, Jeroen Kalkman, and Ad Lagendijk for discussions. This work was supported by the Netherlands Foundation "Fundamenteel Onderzoek der Materie (FOM)" and the "Nederlandse Organisatie voor Wetenschappelijk Onderzoek (NWO)," and is part of an industrial partnership program between Philips and FOM.

${ }^{1}$ J. Wang, M. K. Gudiksen, X. Duan, Y. Cui, and C. M. Lieber, Science 293, 1455 (2001).

${ }^{2}$ H. Pettersson, J. Trägardh, A. I. Persson, L. Landin, D. Hessman, and L. Samuelson, Nano Lett. 6, 229 (2006).

${ }^{3}$ L. K. van Vugt, S. J. Veen, E. P. A. M. Bakkers, A. L. Roest, and D. Vanmaekelbergh, J. Am. Chem. Soc. 127, 12357 (2005).

${ }^{4}$ O. L. Muskens, M. H. M. van Weert, M. T. Borgström, E. P. A. M. Bakkers, and J. Gómez Rivas (unpublished).

${ }^{5}$ W. A. de Heer, W. S. Bacsa, A. Chatelain, T. Gerfin, R. Humphrey-Baker, L. Forro, and D. Ugarte, Science 268, 845 (1995).

${ }^{6}$ F. Genereux, S. W. Leonard, H. M. Van Driel, A. Birner, and U. Gösele, Phys. Rev. B 63, 161101 (2001).

${ }^{7}$ N. Künzner, D. Kovalev, J. Diener, E. Gross, V. Y. Timoshenko, G. Polisski, F. Koch, and M. Fujii, Opt. Lett. 26, 1265 (2001).

${ }^{8}$ M. L. Markham, J. J. Baumberg, D. C. Smith, X. Li, T. Gabriel, G. S. Attard, and I. Nandhakumar, Appl. Phys. Lett. 86, 011912 (2005).

${ }^{9}$ V. S.-Y. Lin, K. Motesharei, K. S. Dancil, M. J. Sailor, and M. R. Ghadiri, Science 278, 840 (1997).

${ }^{10}$ Z. Qi, I. Honma, and H. Zhou, Appl. Phys. Lett. 88, 053503 (2006).

${ }^{11}$ W. U. Wang, C. Chen, K. Lin, Y. Fang, and C. M. Lieber, Proc. Natl. Acad. Sci. U.S.A. 102, 3208 (2005).

${ }^{12}$ M. J. A. de Dood, W. T. M. Irvine, and D. Bouwmeester, Phys. Rev. Lett. 93, 040504 (2005).

${ }^{13}$ M. Kotlyar, L. Bolla, M. Midrio, L. O'Faolain, and T. Krauss, Opt. Express 13, 5040 (2005).

${ }^{14}$ D. Artigas and L. Torner, Phys. Rev. Lett. 94, 013901 (2005).

${ }^{15}$ E. D. Palik, Handbook of Optical Constants of Solids, 1st ed. (Academic, Orlando, 1985), Vol. 1, pp. 445-449.

${ }^{16}$ R. S. Wagner and W. C. Ellis, Appl. Phys. Lett. 4, 89 (1964).

${ }^{17}$ W. L. Bragg and A. B. Pippard, Acta Crystallogr. 6, 865 (1953).

${ }^{18}$ O. Levy and D. Stroud, Phys. Rev. B 56, 8035 (1997).

${ }^{19}$ A. Kirchner, K. Busch, and C. M. Soukoulis, Phys. Rev. B 57, 277 (1998).

${ }^{20}$ S. L. Diedenhofen, O. L. Muskens, M. T. Borgström, E. P. A. M. Bakkers, and J. Gómez Rivas (unpublished). 